\title{
Importancia de la tiroglobulina sérica preablativa en la predicción de sobrevida libre de enfermedad en cáncer diferenciado de tiroides
}

\author{
RENÉ E. DÍAZ ${ }^{1}$, JESÚS VÉLIZ ${ }^{1,2}$, NELSON WOHLLK ${ }^{1,2,3}$
}

Sección de Endocrinología, Hospital del Salvador Santiago, Chile

${ }^{2}$ Facultad de Medicina

Universidad de Chile

División Oriente, Santiago,

Chile

${ }^{3}$ Instituto de Estudios Médicos Avanzados, Santiago, Chile.

Empleando el formulario universal del ICMJE, los autores declararon no tener conflictos de intereses.

Recibido el 27 de agosto de 2013, aceptado el 21 de noviembre de 2013

Correspondencia a: Dr. René E. Díaz. Avenida Salvador 364, Providencia, Santiago. E-mail: diaztorres77@ gmail.com

\section{Preablative serum thyroglobulin as predictor of disease-free survival in differentiated thyroid cancer}

Background: Serum thyroglobulin (sTg) is an excellent marker of persistence or recurrence of disease in differentiated thyroid cancer (DTC), however its role as prognostic factor has not been fully established. Aim: To assess the value of the preablative thyroglobulin ( $p \mathrm{Tg}$ ) as predictor of disease-free survival in DTC. Patients and Methods: Retrospective study of 104 patients with low and intermediate risk DTC subjected to total thyroidectomy and ${ }^{131}$ iodine ablation. TSH, pTg and thyroglobulin antibodies (AbTg) were determined by chemiluminescence. Patients with distant metastases or presence of $\mathrm{AbTg}$ were excluded. Results were analyzed using receiving operating characteristic (ROC) curves. Results: During the $40 \pm 29$ months of follow-up (range 6-132), 14 of 104 (13\%) patients had recurrence of disease. $p \mathrm{Tg}$ was an independent indicator to predict disease-free survival. Using a pTg cutoff of $<10 \mathrm{ng} / \mathrm{ml}$ the negative predictive value was 99\%, sensitivity 93\%, specificity $82 \%$, positive likelihood ratio (LR) 5.2 and negative LR 0,087. Conclusions: $p$ Tg value is useful as a prognostic marker in predicting disease-free survival in DTC patients with low or intermediate risk of recurrence.

(Rev Med Chile 2013; 141: 1506-1511)

Key words: Prognosis; thyroglobulin; Thyroid neoplasms.
E 1 cáncer de tiroides comprende alrededor de $1 \%$ de todos los cánceres y abarca más de $90 \%$ de las neoplasias malignas de origen endocrino. El cáncer diferenciado de tiroides del epitelio folicular (CDT) es el más frecuente (90\%) siendo sus principales tipos histológicos: papilar (85\%), folicular (10\%) y de células de Hürthle $(3 \%)^{1}$.

En general, el CDT es de buen pronóstico de sobrevida, pero con una recurrencia global de hasta 30\%, existiendo casos descritos de recurrencia después de 30 años de seguimiento, principalmente en pacientes con subtipos histológicos más agresivos y enfermedad más extensa (grandes tumores, compromiso de cápsula tiroidea y metás- tasis ganglionares cervicales) ${ }^{2-4}$. Por esta razón, los pacientes requieren seguimiento prolongado una vez realizada la tiroidectomía total (TT) o casi total y en casos seleccionados ablación con ${ }^{131} \mathrm{I}$.

La tiroglobulina ( $\mathrm{Tg}$ ) es una gran glicoproteína $(\sim 660 \mathrm{kDa})$ producida por las células foliculares tiroideas y almacenada en el coloide. La glándula tiroides constituye la única fuente de $\mathrm{Tg}$, de manera que si un paciente con CDT es sometido a TT y ablación del remanente con ${ }^{131} \mathrm{I}$, su nivel de Tg sérica (sTg) durante el seguimiento, teóricamente debiera ser indetectable en ausencia de anticuerpos antitiroglobulina $(\mathrm{AcTg})^{5}$. Esta característica constituye la base para el uso de sTg como marcador tumoral en el CDT; por el contra- 
rio, un nivel detectable es un excelente marcador de persistencia o recurrencia de enfermedad. Sin embargo, su rol como factor pronóstico cuando se mide sTg preablativa (pTg) (inmediatamente previo a la administración de ${ }^{131} \mathrm{I}$ ), no ha sido completamente establecido ya que un remanente tiroideo postquirúrgico podría contribuir de forma variable a incrementar su nivel sérico. Algunos autores consideran que sólo valores postablativos de sTg serían un marcador tumoral confiable, ya que la tiroglobulina producida por el remanente tiroideo es indistinguible de aquella producida por las células tumorales y esta fue la postura que por años se impuso, relegando a la pTg a un nivel secundario como elemento predictor en cáncer de tiroides ${ }^{6}$. Independientemente de lo anterior, los estudios han sido consistentes en mostrar que los pacientes que presentan recurrencia o persistencia de enfermedad tienen valores de pTg más elevados que aquellos que se mantienen libres de enfermedad ${ }^{7}$.

Las guías clínicas otorgan distinta relevancia a la determinación de pTg; en las guías de la American Thyroid Association (ATA), se menciona que valores bajos de $\mathrm{pTg}$ tienen un excelente valor predictivo negativo (VPN) para enfermedad residual, sin especificar punto de corte ni incluirla en alguna de las recomendaciones; por el contrario la Sociedad Latinoamericana de Tiroides (LATS) sugiere su determinación en ausencia de administración de hormona tiroidea, otorgándole un alto valor predictivo positivo (VPP) y el consenso europeo recomienda su medición, incluso cuando la estimulación ha sido realizada con TSH recombinante humana (rhTSH), señalando que valores bajos se asocian a un pronóstico favorable ${ }^{1,8,9}$.

Presentamos un estudio diseñado para evaluar el valor de la pTg como predictor de sobrevida libre de enfermedad en CDT en una población hospitalaria chilena.

\section{Pacientes y Métodos}

Este estudio se basó en los registros retrospectivos de pacientes portadores de CDT controlados en el policlínico de Endocrinología del Hospital del Salvador, Santiago, Chile entre los años 2000 y 2010.

En nuestro estudio se incluyeron todos los pacientes con diagnóstico histológico de CDT (papilar y folicular) tratados con TT y ablación con ${ }^{131}$ I que tuvieron medición de tiroglobulina sérica estimulada (con TSH > $35 \mathrm{mIU} / \mathrm{L}$ ) en el momento previo a la administración de ${ }^{131} \mathrm{I}$, además de seguimiento de al menos un año posterior a la ablación,

Se excluyeron pacientes con carcinomas menores a $1 \mathrm{~cm}$ unifocales, los cuales no son tratados con ${ }^{131}$ I en nuestro centro, pacientes con presencia de AcTg (cualquier valor sobre la sensibilidad analítica fue considerado positivo) y aquellos que tenían evidencia imagenológica de metástasis a distancia al momento del tratamiento con ${ }^{131} \mathrm{I}$.

De 200 pacientes con CDT se excluyeron 56 microcarcinomas, 8 pacientes fueron excluidos por presencia de metástasis al momento del diagnóstico y 32 por presencia de AcTg, quedando 104 pacientes disponibles para el análisis.

Todos los pacientes luego del tratamiento con ${ }^{131}$ I fueron seguidos de acuerdo a las recomendaciones ATA (rastreo sistémico post tratamiento con ${ }^{131} \mathrm{I}$, seguimiento clínico, TSH, T4 libre, ultrasonografía cervical, medición de sTg y AcTg y en casos seleccionados rastreo sistémico con dosis bajas de $\left.{ }^{131} \mathrm{I}\right)^{1}$.

Enfermedad persistente o recurrente se definió por niveles detectables de sTg asociado a valores elevados de Tg en el lavado de la aguja de punción de adenopatías cervicales, confirmación histológica de metástasis ganglionares o evidencia imagenológica de metástasis a distancia.

Sobrevida libre de enfermedad se definió como ausencia de enfermedad estructural (ausencia imagenológica o histopatológica de recidiva) y sTg suprimida $<1 \mathrm{ng} / \mathrm{ml}$ y sTg estimulada con suspensión de hormona tiroidea $<2 \mathrm{ng} / \mathrm{ml}$, en ausencia de $\mathrm{AcTg}^{10}$.

Los niveles de TSH, T4 libre, sTg y AcTg fueron determinados a través de un ensayo inmunométrico (immulite, DPC-Siemens) con una sensibilidad funcional y analítica de $0,36 \mathrm{ng} / \mathrm{mL}$ y $0,2 \mathrm{ng} / \mathrm{mL}$ respectivamente para el ensayo de sTg, y de $20 \mathrm{UI} /$ $\mathrm{mL}$ y $18 \mathrm{UI} / \mathrm{mL}$ respectivamente para el ensayo de AcTg.

Con respecto al análisis estadístico, las variables continuas se describieron mediante promedio y desviación estándar, se compararon por condición de recidiva mediante el test de Mann-Whitney. Las variables categóricas se describieron mediante sus frecuencias y se asociaron con la condición de recidiva mediante el test exacto de Fisher. La discri- 
minación de la condición de recidiva mediante la tiroglobulina se realizó con regresión logística, la capacidad de discriminación se midió con el área bajo la curva ROC.

\section{Resultados}

Las características de los pacientes se resumen en la Tabla 1.

La mayoría de los pacientes correspondieron a cáncer papilar y todos fueron sometidos a TT y recibieron ${ }^{131}$ I en dosis promedio de $121,8 \pm 26,98$ mCi (rango 50-150), realizándose rastreo sistémico posterior al tratamiento con ${ }^{131} \mathrm{I}$ en todos ellos. No se dispuso de este examen en 7 pacientes. No hubo relación entre recidiva y captación fuera del lecho tiroideo.

Tabla 1. Características de pacientes con cáncer diferenciado de tiroides

\begin{tabular}{|c|c|}
\hline Característica & $\begin{array}{c}\text { Porcentaje de } \\
\text { pacientes (n/N) } \\
\text { o media } \pm \text { DE }\end{array}$ \\
\hline Sexo femenino & $84,6 \%(88 / 104)$ \\
\hline Edad (años) & $\begin{array}{r}49,97 \pm 14,31 \\
\text { (rango 20-78) }\end{array}$ \\
\hline $\begin{array}{l}\text { Histología } \\
\text { Papilar } \\
\text { Papilar variante folicular } \\
\text { Folicular } \\
\text { Carcinoma de células de Hürthle } \\
\text { Insular }\end{array}$ & $\begin{array}{l}72,12 \%(75 / 104) \\
12,5 \%(13 / 104) \\
9,62 \%(10 / 104) \\
4,81 \%(5 / 104) \\
0,96 \%(1 / 104)\end{array}$ \\
\hline Dosis ${ }^{131}$ I (mCi) & $\begin{array}{l}121,8 \pm 26,98 \\
\text { (rango 50-100) }\end{array}$ \\
\hline $\begin{array}{l}\text { Estadío TNM } \\
\text { (AJCC, Sixth Edition 2010) } \\
\text { Estadío I } \\
\text { Estadío II } \\
\text { Estadío III } \\
\text { Estadío IVA }\end{array}$ & $\begin{array}{r}56,73 \%(59 / 104) \\
9,62 \%(10 / 104) \\
14,42 \%(15 / 104) \\
19,23 \%(20 / 104)\end{array}$ \\
\hline $\begin{array}{l}\text { sTg preablativa } \\
<0,2 \mathrm{ng} / \mathrm{ml} \\
0,2-10 \mathrm{ng} / \mathrm{ml} \\
\geq 10 \mathrm{ng} / \mathrm{ml}\end{array}$ & $\begin{array}{c}6,7 \%(7 / 104) \\
67,3 \%(70 / 104) \\
26 \%(27 / 104)\end{array}$ \\
\hline $\begin{array}{l}\text { Rastreo sistémico post }{ }^{131} \text { | } \\
\text { Negativo } \\
\text { Captación sólo lecho tiroideo } \\
\text { Captación fuera del lecho tiroideo } \\
\text { Captación extracervical patológica }\end{array}$ & $\begin{array}{c}6,2 \%(6 / 97) \\
62,9 \%(61 / 97) \\
30,9 \%(30 / 97) \\
0 \%(0 / 97)\end{array}$ \\
\hline Recidiva & $13,46 \%(14 / 104)$ \\
\hline
\end{tabular}

Poco más de la mitad de los pacientes fueron clasificados como estadío I TNM.

El valor promedio de pTg fue de 15,42 \pm $39,55 \mathrm{ng} / \mathrm{mL}$ (rango $<0,2-315 \mathrm{ng} / \mathrm{mL}$ ), siendo indetectable en 7 de los 104 pacientes $(6,73 \%)$ y entre 0,2 y $6,8 \mathrm{ng} / \mathrm{mL}$ en 58 pacientes $(55,77 \%)$, es importante recalcar que nuestro estudio excluyó a pacientes con metástasis a distancia al momento de la medición de pTg. El seguimiento se realizó de acuerdo a lo antes señalado.

Durante los 39,98 $\pm 29,04$ meses de seguimiento (rango 6-132), 14 de $104(13,46 \%)$ pacientes presentaron recurrencia de enfermedad, en 13 de ellos la recidiva fue ganglionar cervical y un paciente portador de cáncer folicular presentó una metástasis ósea en pelvis. La presencia de persistencia/recidiva se constató a los $24 \pm 18,38$ meses post cirugía, con un rango de 6-60 meses ( 2 pacientes presentaron persistencia/recidiva de enfermedad precoz, detectada a los 6 meses).

De los 90 pacientes considerados libres de enfermedad se realizó sTg estimulada en 71 pacientes, siendo $<2 \mathrm{ng} / \mathrm{mL}$ en 59 pacientes, $2-10$ $\mathrm{ng} / \mathrm{mL}$ en 7 pacientes $\mathrm{y}>10 \mathrm{ng} / \mathrm{mL}$ en 5 pacientes. Con respecto al grupo que presenta sTg 2-10 ng/ $\mathrm{mL}$, todos ellos se encuentran libre de enfermedad estructural al momento del estudio, pero sabemos que tienen un riesgo de recurrencia que puede alcanzar $20 \%{ }^{10}$. El grupo con sTg $>10 \mathrm{ng} / \mathrm{mL}$ poseen rastreo sistémico post ${ }^{131}$ I y ultrasonografía cervical negativa; en estos 5 pacientes no se logró identificar lesión durante seguimiento prolongado, incluido 18-FDG PET-CT. Todos estos pacientes actualmente tienen sTg $<1 \mathrm{ng} / \mathrm{mL}$ bajo tratamiento con levotiroxina y fueron considerados libres de enfermedad estructural para efectos del análisis estadístico.

A 19 pacientes no se les realizó sTg estimulada por ser considerados de bajo riesgo de recidiva; todos ellos tienen sTg bajo supresión de TSH $<1$ $\mathrm{ng} / \mathrm{mL}, 7$ de ellos tienen 24 meses de seguimiento, 7 de ellos 36 meses y los otros 5 pacientes tienen seguimiento prolongado de hasta 11 años, todos sin evidencia de enfermedad clínica, bioquímica ni imagenológica.

Los valores de pTg en los pacientes con y sin recurrencia de enfermedad fueron de 73,05 \pm $88,18 \mathrm{ng} / \mathrm{mL}$ y $6,45 \pm 8,4 \mathrm{ng} / \mathrm{ml}$ respectivamente $(\mathrm{p}<0,0001)$.

La pTg fue un indicador independiente para predecir sobrevida libre de enfermedad (valor de 


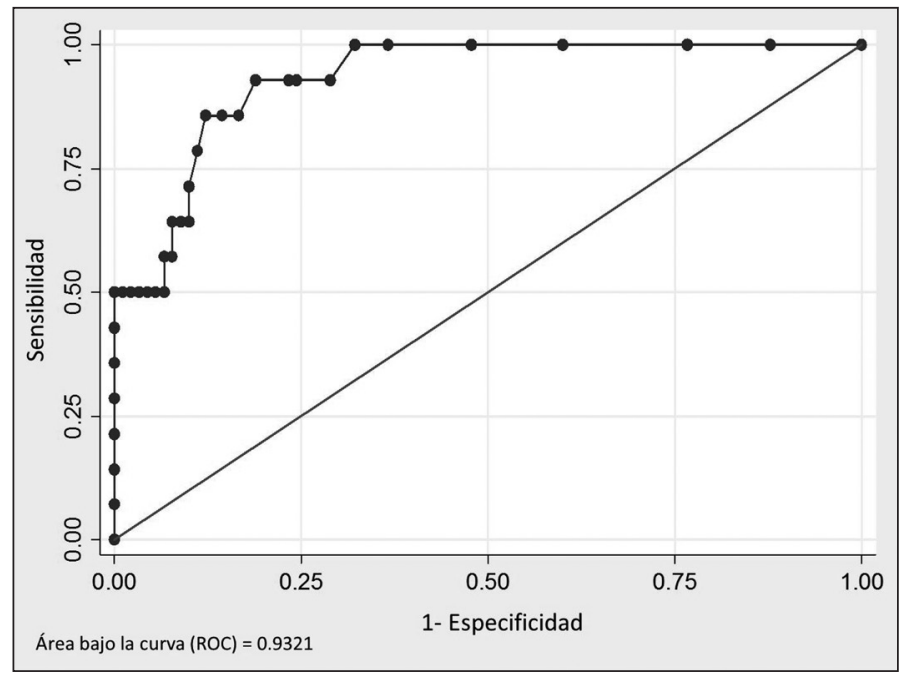

Figura 1. Curva ROC para valor de corte $\mathrm{pTg}$ de $<10 \mathrm{ng} / \mathrm{mL}$ (sensibilidad 92,86\%, especificidad $82,22 \%)$ corte pTg de $<10 \mathrm{ng} / \mathrm{mL}$ ), VPN 98,65\%, sensibilidad 92,86\%, especificidad 82,22\%, LR (+) 5,22 y LR (-) 0,0869 (Figura 1).

Sólo un paciente recidivó con $\mathrm{pTg}<10 \mathrm{ng} / \mathrm{mL}$ ( $6,3 \mathrm{ng} / \mathrm{mL}$ en ausencia de AcTg), el cual presento recidiva ganglionar a los 6 meses postcirugía.

\section{Discusión}

En patología neoplásica existe una búsqueda permanente de marcadores precoces de persistencia y progresión de enfermedad y el CDT no es la excepción, más aun considerando que en algunas ocasiones los primeros indicios de enfermedad persistente aparecen tardíamente ${ }^{4}$.

Como se señaló en la introducción, en el desarrollo de marcadores en CDT el valor de pTg ha tenido un rol incierto, no siendo considerado hasta los últimos años, principalmente por el hecho que remanentes tiroideos postquirúrgicos pueden contribuir de forma variable a incrementar dichos niveles.

El manejo de los pacientes portadores de CDT ha cambiado en los últimos años, pasando de un esquema de tratamiento estandarizado para todos los pacientes a un enfoque basado en la categorización del riesgo de recurrencia, que incluye la respuesta al tratamiento inicial. Dos tercios de los pacientes portadores de CDT pertenecen al grupo de bajo riesgo de recurrencia, siendo necesario identificar elementos que puedan ser utilizados como predictores y que permitan adaptar el tratamiento y seguimiento de los pacientes de acuerdo a su riesgo individual de recurrencia/persistencia de enfermedad. En este contexto la determinación de pTg adquiere gran relevancia ${ }^{10}$.

Se ha intentado establecer valores de corte de pTg para predecir recurrencia o persistencia de enfermedad, pero los VPP obtenidos han sido muy variables; esto ha sido atribuido a la presencia del remanente tiroideo, el cual mantiene su capacidad de producir Tg previo a la ablación con ${ }^{131} \mathrm{I}^{11}$.

Existen pocos estudios que se han enfocado en analizar el VPN de la pTg en pacientes con ausencia de metástasis a distancia, esto es, su capacidad de predecir sobrevida libre de enfermedad. $\mathrm{Al}$ incluir pacientes con metástasis a distancia, los valores de $\mathrm{pTg}$ se elevan sustancialmente y además son pacientes que ya tienen persistencia de enfermedad.

En un trabajo retrospectivo, Giovanella y cols. analizaron 156 pacientes a 12 meses, obteniendo un VPN y VPP de $99 \%$ y $51 \%$ respectivamente con un punto de corte de pTg de 4,5 ng/mL. Piccardo y cols., en el año 2010 evaluaron 169 pacientes con seguimiento de 2 años, obteniendo un VPN de $100 \%$ para un punto de corte de pTg de $10 \mathrm{ng} / \mathrm{mL}^{12,13}$. Estos serían pacientes en los que eventualmente podría no ser necesario realizar sTg estimulada durante el seguimiento, considerando el bajo riesgo de recurrencia que le otorgaría el tener una pTg baja. 
Tiroglobulina preablativa en cáncer de tiroides - R. E. Díaz et al

Un metaanálisis publicado en el año 2012 incluyó 3.947 pacientes de 15 estudios publicados entre los años 1996 y 2011; dentro de estos estudios se incluyeron pacientes con metástasis a distancia e incluso uno de los trabajos analizados incluyó pacientes con presencia de AcTg, mostrando un VPN de $94,2 \%$ para un punto de corte de pTg de $<10 \mathrm{ng} / \mathrm{ml}^{14}$.

Nuestro estudio, realizado en una población de pacientes hospitalarios, reafirma que la $\mathrm{pTg}$ es un excelente predictor de sobrevida libre de enfermedad, con un punto de corte $<10 \mathrm{ng} / \mathrm{mL}$, LR (-) 0,0869, VPN 98,65\%, en un seguimiento a $39,98 \pm 29,04$ meses, lo cual le otorga a esta prueba una gran potencia diagnóstica.

Cabe consignar que en nuestro estudio se excluyeron pacientes con metástasis a distancia, es decir, se trataba de pacientes de riesgo bajo e intermedio, que es precisamente donde la pTg tendría mayor utilidad como factor pronóstico. Setenta y cuatro por ciento de nuestros pacientes tiene $\mathrm{pTg}<10 \mathrm{ng} / \mathrm{mL}$, lo cual le otorga a este grupo un excelente pronóstico y permite adaptar su seguimiento, evitando exámenes y controles innecesarios. La pTg tuvo un pobre rendimiento en predecir recurrencia $(\mathrm{LR}(+) 5,22$ para $\mathrm{Tg}>10$ $\mathrm{ng} / \mathrm{mL}$ ), lo cual está en concordancia con lo que han demostrado otros autores ${ }^{11}$.

En los últimos años la pTg ha sido evaluada como un elemento más para definir aquel subgrupo de pacientes en los cuales podría no ser necesario realizar ablación con ${ }^{131} \mathrm{I}$, postulándose que pacientes con $\mathrm{pTg} \leq 1 \mathrm{ng} / \mathrm{ml}$ y con AcTg negativos podrían manejarse sólo con cirugía, ya que la ablación con ${ }^{131}$ I no conllevaría un beneficio mayor respecto al pronóstico y sobrevida libre de enfermedad en estos pacientes, lo cual incluso ha sido recomendado por la versión 2013 de la guía $\mathrm{NCCN}^{3,15-17}$.

Cabe consignar que en nuestros pacientes los niveles de pTg fueron obtenidos bajo suspensión de levotiroxina, ya que los tomados con estímulo de rhTSH tienden a ser más bajos y no está claramente definido en qué momento deben ser tomados (tercer o quinto día).

En conclusión, los valores de pTg son de utilidad como marcador pronóstico por si solo de sobrevida libre de enfermedad en pacientes con riesgo de recurrencia bajo e intermedio, con un excelente VPN para un punto de corte $<10 \mathrm{ng} / \mathrm{mL}$ en población chilena hospitalaria y en base a esto puede ser utilizado como un elemento más en el momento de definir el tratamiento y seguimiento de pacientes con CDT.

Agradecimientos: Al Dr. Jorge Sapunar y al Sr. Gabriel Cavada por su colaboración en el análisis estadístico de los datos.

\section{Referencias}

1. Cooper DS, Doherty GM, Haugen BR, Kloos RT, Lee SL, Mandel SJ et al. Revised American Thyroid Association management guidelines for patients with thyroid nodules and differentiated thyroid cancer. Thyroid 2009; 19 (11): 1167-214.

2. Schlumberger M, Sherman SI. Approach to the patient with advanced differentiated thyroid cancer. Eur J Endocrinol 2012; 166 (1): 5-11.

3. NCCN Clinical Practice Guidelines in Oncology, Thyroid carcinoma v2.2013. Disponible en http://www. nccn.org/professionals/physician_gls/f_guidelines.asp (consultado el 01 de agosto de 2013).

4. Mazzaferri EL, Kloos RT. Current approaches to primary therapy for papillary and follicular thyroid cancer. J Clin Endocrinol Metab 2001; 86 (4): 1447-63.

5. Demers LM, Spencer CA. Laboratory Medicine Practice Guidelines: Laboratory Support for the Diagnosis and Monitoring of Thyroid Disease. eds (2002) Washington, DC: National Academy of Clinical Biochemistry. Disponible en www.nacb.org (consultado el 01 de agosto de 2013).

6. Mazzaferri EL, Robbins RJ, Spencer CA, Braverman LE, Pacini F, Wartofsky L, et al. A consensus report of the role of serum thyroglobulin as a monitoring method for low-risk patients with papillary thyroid carcinoma. J Clin Endocrinol Metab 2003; 88 (4): 1433-41.

7. Heemstra KA, Liu YY, Stokkel M, Kievit J, Corssmit E, Pereira AM, et al. Serum thyroglobulin concentrations predict disease-free remission and death in differentiated thyroid carcinoma. Clin Endocrinol (Oxf) 2007; 66 (1): 58-64.

8. Pitoia F, Ward L, Wohllk N, Friguglietti C, Tomimori E, Gauna A, et al. Recommendations of the Latin American Thyroid Society on diagnosis and management of differentiated thyroid cancer. Arq Bras Endocrinol Metabol 2009; 53 (7): 884-7.

9. Pacini F, Schlumberger M, Dralle H, Elisei R, Smit JW, Wiersinga W. European Thyroid Cancer Taskforce 2006. European consensus for the management of patients with differentiated thyroid cancer of the follicular epithelium. Eur J Endocrinol 2006; 154 (6): 787-803. 
Tiroglobulina preablativa en cáncer de tiroides - R. E. Díaz et al

10. Tuttle RM, Tala H, Shah J, Leboeuf R, Ghossein R, Gonen $\mathrm{M}$, et al. Estimating risk of recurrence in differentiated thyroid cancer after total thyroidectomy and radioactive iodine remnant ablation: using response to therapy variables to modify the initial risk estimates predicted by the new American Thyroid Association staging system. Thyroid 2010; 20 (12): 1341-9.

11. Kim TY, Kim WB, Kim ES, Ryu JS, Yeo JS, Kim SC, et al. Serum thyroglobulin levels at the time of ${ }^{131}$ I remnant ablation just after thyroidectomy are useful for early prediction of clinical recurrence in low-risk patients with differentiated thyroid carcinoma. J Clin Endocrinol Metab 2005; 90 (3): 1440-5.

12. Giovanella L, Ceriani L, Ghelfo A, Keller F. Thyroglobulin assay 4 weeks after thyroidectomy predicts outcome in low-risk papillary thyroid carcinoma. Clin Chem Lab Med 2005; 43 (8): 843-7.

13. Piccardo A, Arecco F, Morbelli S, Bianchi P, Barbera F, Finessi M, et al. Low thyroglobulin concentrations after thyroidectomy increase the prognostic value of undetectable thyroglobulin levels on levothyroxine suppressive treatment in low-risk differentiated thyroid cancer. J
Endocrinol Invest 2010; 33 (2): 83-7.

14. Webb RC, Howard RS, Stojadinovic A, Gaitonde DY, Wallace MK, Ahmed J, et al. The utility of serum thyroglobulin measurement at the time of remnant ablation for predicting disease-free status in patients with differentiated thyroid cancer: a meta-analysis involving 3947 patients. J Clin Endocrinol Metab 2012; 97 (8): 2754-63.

15. Vaisman A, Orlov S, Yip J, Hu C, Lim T, Dowar M, et al. Application Of Post-Surgical Stimulated Thyroglobulin For Radioiodine Remnant Ablation Selection In LowRisk Papillary Thyroid Carcinoma. Head Neck 2010; 32 (6): 689-98.

16. Rosario PW, Xavier AC, Calsolari MR. Value of postoperative thyroglobulin and ultrasonography for the indication of ablation and ${ }^{131}$ I activity in patients with thyroid cancer and low risk of recurrence. Thyroid 2011; 21 (1): 49-53.

17. Nascimento C, Borget I, Al Ghuzlan A, Deandreis D, Chami L, Travagli JP, et al. Persistent disease and recurrence in differentiated thyroid cancer patients with undetectable postoperative stimulated thyroglobulin level. Endocr Relat Cancer 2011; 18 (2): R29-40. 